\title{
LOS CÍCLIDOS (PISCES: CICHLIDAE) EN COLOMBIA: INTRODUCCIONES, TRASPLANTES Y REPOBLACIONES
}

\author{
FRANCISCO DE PAULA GUTIÉRREZ-BONILLA ${ }^{1}$ \\ RICARDO ÁLVAREZ-LEÓN ${ }^{2}$ \\ francisco.gutierrez@utadeo.edu.co, alvarez_leon@yahoo.com
}

Manizales, 2011-07-16 (Rev. 2011-08-09)

\section{RESUMEN}

Se incluye el estado actual de las especies de la familia Cichlidae introducidas a Colombia, con especial énfasis en las tilapias, analizando la situación de su distribución, el manejo, los impactos y la situación de estas actividades ilícitas. El número de introducciones alcanza la cifra de 26 , entre las cuales 12 han sido registradas en hábitats dulceacuícolas y estuarinos. Existe entre estos últimos varios casos de hibridación y trasplantes a diferentes cuencas hidrográficas. Su presencia en las granjas de producción acuícola y desde hace algunos años en las pesquerías, agrava aún más el futuro de las especies nativas.

\section{PALABRAS CLAVE:}

Introducciones, trasplantes, repoblación, hibridización, pesquerías, acuicultura, tilapias, ornamentales, Colombia.

\section{CICHLIDS (PISCES: CICHLIDAE) IN COLOMBIA: INTRODUCTIONS, TRANSPLANTS AND RESTOCK}

\section{ABSTRACT}

This paper includes the current status of the Cichlidae family species introduced in Colombia, with special emphasis on tilapia,. analyzing the situation of its distribution, management, and the impact and status of these illegal activities. The number of intriductions is up to 26 species, among which 12 have been found in freshwater and estuarine habitats. There are several hybridization and transplant cases to different hydrographic basins. Their presence in the aquaculture production farms and,in the fishing industry in recent years, worsens even more the future of native species.

\section{KEY WORDS:}

Introduction, transplants, hybridizations, fishing industry, aquaculture, tilapias, ornaments fish, Colombia.

\section{INTRODUCCIÓN}

Las introducciones, poseen 4 dimensiones en su manejo: (1) la cultural: pues el manejo de las especies exóticas tiene que ver con los valores que las personas tienen, lo que encuentran bello, o permisible; (2) la educación: ¿qué tanto conocen las personas sobre el tema?; (3) la salud: de donde se desprende la posibilidad de patógenos y (4) la dimensión filosófica: que hace reflexionar sobre los cambios que estamos provocando y sí estamos dejando a las nuevas generaciones, mejores o peores ecosistemas. 
Introducir especies no se corresponde con la distribución natural de las especies, ni con la prevención que se debe tener respecto al cuidado de la diversidad biológica, más aún cuando tales acciones ocurren en ecosistemas acuáticos, de donde será extremadamente difícil o imposible su erradicación.

Introducir especies es un primer tema, pero un segundo es el trasplante (traslado de una especie nativa local de una cuenca hidrográfica a otra en el mismo país). En Colombia, normativamente el trasplante (Código de los Recursos Naturales y de Protección del Ambiente -Decreto Ley 2811 de 1974-; Reglamentario en Materia de Fauna Silvestre -Decreto 1608 de 1978-) no se considera una introducción, pero biológicamente lo es y en ocasiones su impacto puede llegar a ser igual o mayor (Gutiérrez-Bonilla, 2005, 2010).

A escala global la diversidad biológica catalogada es cercana a 1,8 millones de especies. En este contexto, la diversidad en aguas continentales es muy alta comparada con la de otros ecosistemas. Los hábitats de aguas continentales cubren menos del $1 \%$ de la superficie del planeta, y albergan más del $25 \%$ de todos los vertebrados descritos, más de 126.000 de las especies conocidas de animales, y aproximadamente 2.600 plantas macrófitas. Calculándose que hay unas 27.400 especies de peces, moluscos, cangrejos, libélulas y plantas de agua dulce. De estas, hasta la fecha solo 6.000 se han evaluado, quedando por hacerlo sobre más de 21.000 especies. Estos ecosistemas proporcionan bienes y servicios ambientalmente importantes, calculándose que su valor es de 70 billones de dólares -cifra equivalente al PIB de algunos países del tercio superior de las economías mundiales- (IUCN, 2008).

Los hechos demuestran que nunca nos hemos preguntado: ¿Qué sucede cuando se introduce una especie en un ecosistema al cual no tendría acceso en forma natural? ¿Son los ecosistemas flexibles y toleran el cambio o se pueden dar grandes repercusiones, provocando daños permanentes? ¿Algo especial se perderá para siempre? ¿Es eso importante? Esas mismas preguntas son válidas cuando hablamos de Organismos Vivos Modificados (OVM), o de Organismos Genéticamente Modificados (OGM) usualmente utilizados sin el debido biorrigor y análisis previos, quedando en la mayoría de los casos sujetos solamente a la bondad de las premisas económicas, para así autorizar su utilización (Devlin et al., 2007).

La globalización de las introducciones y la aparición de especies invasivas producto de las impensadas introducciones se ha incrementando, generando impactos negativos irreversibles a la biodiversidad, a los sistemas agrícolas y de manera directa e indirecta a la salud humana, que se están magnificando por los cambios globales a nivel físico y biótico, originando alteraciones en los ecosistemas y en la distribución de las poblaciones (Kingston \& Waldren, 2003; Lee et al., 2003). La introducción de especies ha sido identificada como uno de los riesgos más críticos a los que están expuestos los ecosistemas, las comunidades, las poblaciones y la biodiversidad en general (Hopkins, 2001). Así, la introducción de especies está asociada en un $54 \%$ con la extinción de la fauna acuática nativa mundial (Harrison \& Stiassny, 1999), en un $70 \%$ para el caso de los peces de Norteamérica (Lassuy, 2002) y en un $60 \%$ para el caso de México y algunos países africanos. Caso extremo de las introducciones mal planificadas y con propósitos estrictamente económicos, fue lo ocurrido en el Lago Tanganika (compartido por cuatro países: Burundi, República Democrática del Congo, Tanzania y Zambia) donde se extinguieron 200 especies endémicas, en el Lago Malawi (Mozambique, Malaui y Tanzania) 300 especies y en el Lago Victoria (Uganda, Tanzania y Kenia) 220 especies (Contreras \& Escalante, 1984; Contreras-Balderas, 1999; Contreras, 2002). 
La "contaminación biológica" así denominada el ingreso de especies exóticas (Moyle \& Leidy, 1992; Allan \& Flecker, 1993), fue registrada en ecosistemas continentales y estuarinos, a través de los estudios realizados por la FAO a finales de la década de los setenta al reportar la introducción de 237 especies a aguas continentales en 140 países (Welcomme, 1981). En peces, diez especies fueron las comúnmente introducidas: Carassius auratus, Ctenopharyngodon idella, Cyprinus carpio, Gambusia affinis, Hypophthalmichthys molitrix, $H$. nobilis, Micropterus salmoides, Oncorhynchus mykiss y Oreochromis niloticus. Todo apuntaba al hecho de que el fomento a las pesquerías y a la acuicultura, que poseía un legado de cientos de años a través de las introducciones, los trasplantes y la repoblación, eran y aún son percibidas como acciones económicamente rentables y sin ninguna consideración biológica de sus potenciales impactos negativos. Entre 1940 y 1960, se asumió que hábitats como los embalses, los reservorios requerían nuevas especies y ello reforzó los procesos de introducción y trasplante que luego accidental o voluntariamente pasaron a las cuencas continentales (Hall \& Mills, 2000; Font, 2003). Esta acción se ha acentuado debido a la declinación de la oferta natural de los recursos pesqueros y al desconocimiento biológico de las poblaciones objeto de aprovechamiento, que son de difícil recuperación. Se calcula que los daños causados por especies invasoras ascienden a 1,4 billones de dólares al año y que su control demanda 420.000 millones de dólares (Baptiste et al., 2010). Colombia no se ha quedado atrás, teniendo registradas 581 especies invasoras, incluidas especies de todas las taxas, de las cuales 190 han sido catalogadas como de alto riesgo (Baptiste et al., 2010).

\section{ANTECEDENTES NACIONALES}

En Colombia, las introducciones y los trasplantes con recursos hidrobiológicos y pesqueros han sido realizados por particulares y entidades gubernamentales, sin que para ello se hubiesen desarrollado estudios biológicos previos, para establecer la bondad o no de tales acciones. Sin estos presupuestos, las especies han sido utilizadas por la acuicultura (de fomento, intensiva, extensiva), por la pesca deportiva, como ornamentales y para repoblación o reintroducción.

Los trasplantes deben considerarse en un todo similar a la introducción, por cuanto la población trasplantada nunca estuvo (o no hay prueba de ello) representada en el área donde se le introduce. Además, ofrece los mismos problemas y peligros potenciales que la introducción, pues son elementos adventicios (Lachner et al., 1970). Este hecho tiene notable trascendencia, pues cada ecosistema tiene una dinámica propia, dentro de un equilibrio establecido y así, los elementos adventicios de la misma manera que eventualmente pueden ser exitosos, se convierten en factores adversos de difícil mitigación o erradicación. Biológicamente es válido pensar que si la(s) especie(s) nativa(s), en algún momento de la historia estuvo presente(s) en un ecosistema, no puede suponerse que sea viable su reintroducción, pues las condiciones del equilibrio primigenio no están presentes y los ecosistemas poseen readecuaciones y nuevos balances bioecológicos que previamente deben conocerse y valorarse.

Las decisiones tomadas a este nivel para proceder a las introducciones y los trasplantes, no parecen haberse preguntado: ¿Qué efectos nocivos traerían las especies en el medio natural? (no en confinamiento). AceroSánchez y Hernández-Camacho (1971) expresaron que omitiendo la eventualidad de que la especie introducida o trasplantada pudiese experimentar ciertos cambios significativos en sus hábitos alimenticios, cambios por cierto muy difíciles de prever, establecieron que los principales peligros que conllevaban las introducciones eran: (1) 
imposibilidad de controlar efectivamente su densidad y capacidad de expansión de su área de distribución; (2) ausencia de mecanismos naturales (acción de competidores y depredadores efectivos), que regulen su número; (3) incremento exagerado poblacional que atente contra la estabilidad de la biocenosis y (4) alteración de las cadenas tróficas por la competencia directa o indirecta por alimento con los consumidores primarios nativos, pudiendo llevar a muchas poblaciones a una drástica reducción, o a procesos de extinción. Este peligro es tanto mayor, si se trata de especies muy prolíficas o carnívoras.

Universalmente y frente a la pérdida de poblaciones nativas, quienes apoyan las introducciones han continuado argumentado: "la existencia de nichos ecológicos y niveles tróficos vacíos o vacantes", presunciones biológicas que no son válidas y que Dahl (1958) las desestimó cuando se planteó introducir el bagre pintado (Pseudoplatystoma magdaleniatum) al río Sinú con el argumento de que podía hacerse dada la similitud de las especies con las cuencas del río Magdalena y el San Jorge donde estaba presente. Ramos-Henao)comentando sobre las especies autóctonas de mayor importancia potencial y actual, llego a afirmar que:

\begin{abstract}
"la piscicultura colombiana debería tener como base a los peces nativos, pues la fauna autóctona es muy rica en diversidad de especies, entre las cuales seguramente podrán encontrarse algunas que se presten para el cultivo en estanques agrícolas y comerciales" (Ramos-Henao, 1979, p. 51)).
\end{abstract}

La primera aproximación a las "declaraciones de efecto ambiental", realizadas para autorizar las introducciones en Colombia, se centraron en análisis bibliográficos, o en ensayos ex situ, sin réplicas a mayor a escala, es decir, sin la ejecución de estudios que permitieran tener certeza científica de tales acciones. Este proceder permitió las introducciones, sin tener en cuenta la estructura de la ictiofauna implicada, sus factores bióticos y abióticos favorables, restrictivos y sus requerimientos ecológicos. En otros casos, los estudios fueron realizados con posterioridad a las introducciones, o simplemente no se han realizado.

Colombia tiene una distribución de especies introducidas y trasplantadas, que parece estar originando impactos biológicos aún no precisados, debidos especialmente al impensado proceso de la acuicultura y al repoblamiento con salmónidos (truchas y salmones), cíclidos (tilapias y mojarras) y carácidos (cachamas) que poseen poblaciones de un relativo elevado número de individuos en las cuencas de los ríos Atrato, Cauca, Cesar, Magdalena, Orinoco, San Jorge, Sinú, y principalmente en los departamentos de Atlántico, Bolívar, Boyacá, Cesar, Córdoba, Cundinamarca, Magdalena, Nariño y Valle del Cauca (Otero, 1989; DíazSarmiento \& Álvarez-León, 1998; Álvarez-León \& Rodríguez-Forero, 2000; Álvarez-León et al., 2002; Gutiérrez-Bonilla, 2001, 2005). De todas maneras hay opiniones contrastadas, pues Erazo-Killer (1989) afirmó y lo siguen haciendo muchos autores, que un adecuado control de las poblaciones exóticas puede evitar efectos negativos sobre las nativas; opiniones similares han expresado De longh y Van Zon (1993) que registran para el caso de Tailandia, por lo menos nueve aspectos que han beneficiado al país desde el punto de vista socio-económico, sin que el uso de especies introducidas haya provocado severos impactos ecológicos en términos de deterioro de los ecosistemas acuáticos, salvo casos incidentales. Opiniones que en 2009, podrían ser revaluadas al demostrarse que en por lo menos 52 de los 96 países, en donde han sido introducidos estos cíclidos, se han tenido impactos negativos sobre ecosistemas y poblaciones naturales de especies nativas. Por lo que siempre persistirán dos corrientes en el tratamiento del tema: los beneficios económicos y la inconveniencia biológica, demostrada en el 
hecho de que la introducción de especies es responsable en un $40 \%$ de la pérdida de la diversidad biológica (McNeely et al., 2001; GISP, 2005).

Un inventario preliminar de las especies de cíclidos introducidas a Colombia, muestra a 24 especies, las cuales están siendo destinadas principalmente a la acuicultura y a la acuariología (Tabla 1), y provienen de un amplio rango de la distribución mundial. El conocimiento de especies exóticas tales como las tilapias (Oreochromis hornorum, $\mathrm{O}$. niloticus, Tilapia rendalli) es extenso, gracias a las experiencias realizadas en el ámbito internacional, por lo que son preferentemente utilizadas a nivel nacional para la producción de carne en lugar de las especies nativas como el bocachico (Prochilodus magdalenae), la sabaleta (Brycon henni), las doradas (Brycon moorei, B. sinuensis), la mojarra amarilla (Caquetaia kraussii), la mojarra negra (Caquetaia umbrifera), el tucunaré (Cichla monoculus), el óscar (Astronotus ocellatus) y las cachamas (Colossoma macropomum, Piaractus brachypomus). Sobre estas últimas especies, existen las mejores perspectivas y desde 1980 se desarrollan investigaciones y experiencias muy satisfactorias (Lovshin, 1980; Martínez-Espinosa, 1984), aunque también han pasado al medio natural en ecosistemas continentales (ríos Cauca, Magdalena, San Jorge y Sinú y Ciénaga Grande de Santa Marta).

En 1971, se realizó el "1er. Seminario sobre Piscicultura en Colombia", identificándose las necesidades de investigación en especies nativas y un análisis riguroso respecto a las especies exóticas de interés para ese momento (Cyprinus carpio var. carpio, C. carpio var. specularis, Micropterus salmoides, Salmo spp. y Oreochromis spp.), al trasplante con Cichla monoculus y a las actividades de repoblación, surgiendo recomendaciones muy precisas a ser puestas en práctica, que 40 años después está demostrado no se acataron.

Popma y Villaneda-Jiménez (1977) sustentaron la introducción de O. niloticus, apoyados en los buenos resultados obtenidos en otros países tropicales, argumentando que su ingreso al sistema magdalénico sería un medio de control para otra especie foránea, la Oreochromis mossambicus -para esa fecha ya establecida en esta cuenca-, pues le generaría competencia directa en espacio, alimentación e hibridación.

Popma y Lozano-Díaz (1977a, 1977b, 1977c) realizan las declaraciones de efecto ambiental para el cultivo de $O$. niloticus y Tilapia rendalli en las cuencas Cauca y Magdalena y para el trasplante de C. monoculus como depredador en los cultivos experimentales en las estaciones del Alto y Bajo Magdalena, recomendando medidas para evitar el escape a cuerpos de aguas naturales dado que eran imprevisibles sus impactos.

En 1978, el INDERENA, las universidades de Caldas, Valle, Nacional, Auburn, la Agencia Internacional para el Desarrollo (AID) y el Instituto de Ciencias Naturales (ICN), realizaron un taller para analizar el tema de las introducciones y trasplantes y establecieron: (1) ampliar la zona de utilización para $T$. rendalli al sistema magdalénico, exceptuando la Orinoquia y la Amazonia; (2) no introducir O. niloticus y dar mayor énfasis al estudio y fomento de especies nativas; (3) autorizar el fomento con $T$. rendalli de preferencia en policultivo con especies nativas; (4) esperar los resultados de los estudios que se desarrollaban con C. carpio var. specularis, M. salmoides, Onchorhyncus mykiss y O. mossambicus, para tomar decisiones; (5) utilizar C. monoculus, en los lugares donde ya estaba presente, esto era: Antioquia, Huila, Santander y Valle del Cauca y (6) estudiar de inmediato en la Estación de Repelón, los policultivos de Oreochromis spp., con especies nativas locales predadoras de la Costa Caribe. 
Con ocasión de las cinco reuniones de evaluación e identificación de líneas de acción e investigación prioritaria para la acuicultura, celebradas en Manizales (Simposio sobre Sistemas de Acuicultura para Colombia, 1983), Bogotá D.E. ( $1^{\mathrm{a}}$ y $2^{\mathrm{a}}$ Reunión sobre el Cultivo de la Cachama, 1983 y 1984) y Cúcuta ( $3^{a}$ Reunión sobre el Cultivo de la Cachama, 1985), las de la Red Nacional de Acuicultura (Bogotá, 1987; Gigante, 1988; Calima, 1989; Bogotá, 1990), así como la formulación del Programa Nacional para la Acuicultura en julio de 1985, se establecieron los pasos necesarios para identificar los proyectos prioritarios y orientar en forma óptima los fondos destinados a la investigación, el fomento y a la comercialización, pero también se trazaron líneas de acción respecto a las introducciones y trasplantes, que no fueron implementadas, obrándose en sentido contrario.

\section{LOS REGISTROS PARA CÍCLIDOS}

En la Estación de Piscicultura de la Universidad de Caldas, se realizaron numerosos ensayos con $T$. rendalli tanto en monocultivos como al probar sus efectos en policultivo con especies nativas (Caquetaia kraussii, Prochilodus magdalenae, Rhamdia quelen, "Pimelodus clarias"), trasplantadas (Cichla monoculus) e introducidas (Cyprinus carpio var. specularis, Oreochromis niloticus, Onchorhyncus mykiss) (CEP-UDC, 1973-1983).

Rodríguez-Guerrero y Phelps (1982), ya introducida O. niloticus, determinan la competencia por alimento y espacio, entre esta y 13 especies nativas del Bajo Magdalena, encontrando que había desplazamiento de las nativas. Paredes (1995) catorce años después de introducida la Oreochromis spp., realiza un estudio de su impacto en un policultivo de ocho especies nativas y una exótica (O. niloticus), también en el Bajo Magdalena, y concluye que los riesgos de su introducción a ecosistemas naturales son altos, sin embargo, ya estaba en aguas naturales accidental o voluntariamente.

Vale la pena resaltar que solo a tres especies de cíclidos, se les efectuó el estudio de declaración de efecto ambiental: el tucunaré (C. monoculus), la tilapia herbívora (Tilapia rendalli) y la tilapia nilótica (O. niloticus) (Popma \& Villanada-Jiménez, 1977; Popma \& Lozano-Díaz, 1977a, 1977b, 1977c; Rodríguez-Guerrero \& Phelps, 1982), para justificar su introducción y/o trasplante a diversas cuencas. Sin embargo, los estudios fueron muy superficiales, teóricos y los ensayos en estanques con especies nativas, denotaron la inconveniencia de que estas pasaran en algún momento al medio natural (Álvarez-León \& Rodríguez-Forero, 2000; Álvarez-León et al., 2002).

Garzón (1990) en un análisis de la acuicultura a escala nacional, registró que el manejo de programas de fomento piscícola para 5.700 usuarios, se hacía a través de 40 entidades con el soporte de 31 estaciones piscícolas, 84 extensionistas y concluyó que todo se había implementado sin contar con profesionales y técnicos capacitados en la labor de extensión, asistencia al usuario y sin sopesar el potencial impacto que las especies utilizadas podrían ocasionar una vez dispersadas al medio natural.

Los cíclidos a escala global han gozado de especial preferencia para acciones de introducción, trasplante y repoblación en ecosistemas acuáticos continentales. A su vez, todas estas acciones han conllevado a que en granjas piscícolas, o en laboratorio se desarrollen acciones de hibridación tal como ocurrió en la Florida (USA), en donde fueron producidas por acuaristas o por la industria de peces ornamentales, 
reportándose 20 especies exóticas y 5 híbridas que habían establecido poblaciones reproductivas (Courtenay \& Robins, 1979). Alrededor del mundo, el comercio de peces de acuario representa una de las cinco mayores vías para la introducción, registrándose 150 especies que han invadido los ecosistemas naturales (Ruiz, 1997; Siguan, 2003; Rilov \& Crooks, 2007). Actualmente, en vista de las múltiples y negativas experiencias, estas actividades suelen estar debidamente reguladas internacional y nacionalmente por normas específicas que incluyen aspectos sanitarios y aduaneros, pero su aplicación es parcial o nula.

En Venezuela, está registrado el impacto negativo por la introducción de los cíclidos $O$. mossambicus, $O$. niloticus y Oreochromis spp., que han pasado de los criaderos a aguas naturales en 13 de los 15 Estados, estando ausentes en los Estados Amazonas y Delta Amacuro (Solórzano et al., 2001). En el Lago de Valencia, O. mossambicus se ha convertido en especie dominante y puede estar implicada debido a su característica de depredador, en la casi extinción de Atherinella venezuelae (Tinícalo del Lago de Valencia) pez endémico de esta cuenca (Infante, 1985). Jiménez (1977 citado por Pérez et al., 1997) registra que en la Laguna de los Patos (Cumaná) en 1965, estaban presentes 23 especies nativas de peces y 12 años después, solo diez. Con base en el estudio del contenido estomacal de los cíclidos, se comprobó que tal reducción se debía en gran medida a la depredación sobre las larvas y juveniles de las especies nativas. Valga anotar que hubo una modificación trófica de las especies introducidas que biológicamente no fue previsible, pues normalmente se postula que tales cambios ocurren a largo plazo. Para esa fecha, las especies introducidas habían invadido la cuenca del río Manzanares (que drena directamente al Mar Caribe) y eran dominantes en otras lagunas.

A su vez en Venezuela, han invadido sectores del Golfo de Cariaco y aparecen en las capturas de la pesca marino costera que se desarrolla en el área. Oreochromis spp., está registrada desde 1990 en la cuenca de otro río caribeño, el Tocuyo en el Estado Lara, en el Lago de Maracaibo, en la Bahía del Tablazo, en el sistema del Gran Eneal, en el río Escalante, en áreas adyacentes a los embalses Manuelote y Tulé, en la subcuenca del río Guasare y en la Ciénaga de Los Olivitos, todas localidades del Estado Zulia. O. niloticus está registrada también en embalses que represan las aguas del río Unare y forman parte de la cuenca del Caribe (Estado Anzoátegui) y recién se ha registrado en el río Tuy, principal afluente de la región centro-norte costera de Venezuela (GonzálezOropeza, com. pers.). Tal vez el caso más dramático se refiere a la introducción de C. kraussi en la cuenca del Orinoco (Royero-León \& Lasso-Alcalá, 1992; Señaris \& Lasso-Alcalá, 1993). Esta especie, originaria de las cuencas del río Magdalena (Colombia), Maracaibo y Caribe, e introducida accidentalmente en los llanos inundables del Estado Portuguesa, se incorporó a ambientes naturales tras las crecientes del río (Mago-Leccia, 1978). En la actualidad ha desplazado a los cíclidos nativos de los llanos venezolanos, e incluso a otras especies llaneras, aportando en las lagunas inundables la mayor biomasa (C. A. Lasso-Alcalá, com. pers.).

La cuantificación sobre especies introducidas y trasplantadas en aguas continentales de Colombia, tiene en la década de los 80 registros que empiezan a generar información. Rodríguez-Gómez (1984) registró la introducción de 35 especies de peces (29 ornamentales; seis de consumo) de las cuales 11 estaban en cuerpos de aguas naturales (seis de consumo y cinco ornamentales). COPESCAL (1986) registró que de 1940 a 1986 en Colombia se habían introducido 37 especies, y de estas 27 eran ornamentales. Entre 1997 y 2002, se registraron entre especies, subespecies, variedades, híbridos y formas de cultivo, 162 diferentes tipos de organismos. Los peces fueron 154 y, de estos, 97 eran introducciones y 57 trasplantes, comprendidos en 31 familias de peces y cinco de crustáceos. Las familias con mayor número de especies fueron: 
Cyprinidae con 37, Cichlidae con 35, Belontiidae con 12, Pimelodidae con 11, Characidae con 10, Salmonidae con ocho, Poeciliidae y Serrasalminae con siete. Respecto a los peces introducidos, 93 se registraron en aguas artificiales y 32 en aguas naturales. De los trasplantados, 53 estaban en aguas artificiales y 17 en aguas naturales. Las cuencas hidrográficas con mayor número de especies ícticas introducidas y traslocadas fueron: el Medio Cauca con 90, el Alto Cauca con 89 y el Medio Magdalena con 80. La vertiente con menor "contaminación biológica" fue la del Caribe Sur, con ocho especies. En aguas naturales y respecto a especies introducidas, el Medio Magdalena poseía 15, el Alto Cauca 14 y el Alto Magdalena 13. Por departamentos y considerando especies introducidas y trasplantadas, el Valle del Cauca poseía 91 de 27 familias, Caldas 76 de 10 familias y Antioquia 72 especies de 12 familias (Alvarado-Forero \& Gutiérrez-Bonilla, 1999, 2002; Gutiérrez-Bonilla, 2005, 2010). Se registraron híbridos con introgresiones genéticas de otras especies, y de los mismos no se tiene certeza de que hayan sido colocados en el medio natural (Burbano \& Usaquén, 2003).

En el Lago Nicaragua (Nicaragua) y en Colombia en los embalses de Betania (departamento del Huila) e Hidroprado (departamento del Tolima), la tilapia (Oreochromis spp.) se ha convertido en la especie más abundante (McKaye, 1977; McKaye et al., 1995; Alvarado-Forero, 1998; Alvarado-Forero \& Gutiérrez-Bonilla, 1999; Gutiérrez-Bonilla, 2009) siendo objeto de una intensa actividad de pesca artesanal y acuacultura, que al 2011 representa junto con O. niloticus y O. niloticus var. chitralada las especies objeto de cría y levante en el embalse en donde la producción de cíclidos introducidos (O. niloticus y Oreochromis spp.) aportan en aguas naturales 678 t.año $^{-1}$, a lo que deben sumarse las 12.000 t.año $^{-1}$ de los cultivos en jaulas, que manejan aproximadamente 61,5 millones de individuos (Gutiérrez-Bonilla, 2009).

\section{PISCICULTURA}

Shelton y Smitherman (1984) postulan que las fugas de peces de cultivo son inevitables y Welcomme (1988) a través de sus estudios ha podido determinar que las especies utilizadas en acuicultura eventualmente pasan al medio natural y, por lo tanto, cualquier introducción con fines de cultivo es una adición potencial a la fauna silvestre. De ahí, que en algunos estados de Estados Unidos, esté prohibida la introducción o cultivo de algunas especies y entre ellas las tilapias por su comportamiento agresivo y marcada competencia con especies nativas.

La industria piscícola colombiana la conforman empresas que trabajan principalmente en tilapias (Oreochromis nilotica, O. nilotica var. chitralada, O. spp.), es la más desarrollada, seguida del cultivo de cachamas (Colossoma macropomun, Piaractus brachypomus) y truchas (Oncorhynchus mykiss). La tilapia roja híbrida (Oreochromis spp.) presenta ventajas muy importantes tales como poseer un alto porcentaje de masa muscular, ausencia de espinas intramusculares, crecimiento rápido, alta resistencia a enfermedades y, sobre todo, unas características externas de forma y coloración que han favorecido su creciente demanda en el mercado internacional.

A partir de mediados de la década de los 90 , se ha registrado un incremento en el consumo de estas especies en el país y Colombia se encuentra entre los cuatro principales países productores dentro de América Latina y el Caribe. La explicación a esta diversificación en el cultivo, consiste en el impulso que se le ha dado a nivel de las políticas 
estatales y a la iniciativa de empresarios particulares para involucrar las especies nativas a la acuicultura (Salazar-Ariza, 1999).

Los recientes avances en la valoración de los paquetes tecnológicos completos para tres de las especies más importantes: cachama, trucha y tilapia, permitirán obtener la información técnica y económica básica para hacer de la acuicultura continental una actividad más rentable y sostenible. Especialmente porque dicho trabajo incluye infraestructura física, parámetros técnicos del ciclo de producción, requerimientos de personal, costos fijos y variables de inversión, proyección de ingresos y rentabilidad (Beltrán-Turriago et al., s.f.). Recientemente, sobre la viabilidad y alternativa de producción sostenible, se utilizó en el departamento de Caldas la truchicultura como caso de estudio (LópezMacías et al., 2007).

Para 2002 y 2003, respecto a la producción pesquera nacional, la acuicultura representó el $23,08 \%$ y el $25,65 \%$. En 2002 , aportó 44.177 t.año- $0^{-1}$ y 50.238 t.año $^{-1}$ en 2003 , y dentro de estas cifras, las especie introducidas y/o trasplantadas aportaron a la producción de la acuicultura el $93,19 \%$ y $93,18 \%$ respectivamente $\left(41.172\right.$ t.año $^{-1}, 46.812$ t.año $\left.{ }^{-1}\right)$. Para los años 2002 y 2003, la acuicultura superó a la pesca artesanal continental que lleva más de una década en franco descenso con producciones anuales de 26.500 t.año $^{-1}$. Para los últimos 10 años, la acuicultura ha tenido un promedio anual de producción de 56.793 t.año $^{-1}$ con una máxima producción en 2009 de 77.941 t.año- ${ }^{-1}$ siendo las especies introducidas o trasplantadas el 73,54\% (Robles, 2008; MADR / CCl, 2010). (Figura 1 ). A 2008, la producción pesquera y acuícola alcanzó las 166.000 t y de estas 28.718 t procedían de la pesca continental, por lo que la cifra sigue siendo inferior en 22.913 t (ICA, 2008).

La tilapia roja (Oreochromis spp.) está ampliamente diseminada a nivel nacional a excepción del departamento del Guainía y de manera muy localizada en la cuenca del Amazonas. Dahl (1958), refiriéndose a Colombia, hace 45 años expresó:

\begin{abstract}
"tenemos por fin las especies de tilapias, otros forasteros fracasados en las aguas libres. Estos pececillos en aguas colombianas empiezan a reproducirse, como hemos visto, con mucha frecuencia cuando apenas alcanzan una longitud de unos $5 \mathrm{~cm}$, y llenan las aguas con peces miniaturas no comestibles. En pozos aislados, se pueden utilizar como peces de forraje para el tucunaré y otros peces deportivos, pero por lo menos en las tierras calientes no hay razón para introducir especies exóticas, cuando los ríos y las ciénagas de Colombia abundan precisamente en carácidos pequeños y ciprinodóntidos, excelentes peces de forraje Dahl (1958, p. 4),".
\end{abstract}

\title{
PRODUCCIÓN PESQUERA
}

Buscando comprobar la hipótesis de que la acuicultura es la principal responsable de las introducciones y los trasplantes, el análisis de las estadísticas pesqueras permite determinar que las tilapias comienzan a ser registradas en las estadísticas de desembarco para la cuenca del río Grande de La Magdalena (Magangué) en 1992, con 58 t.año $^{-1}$., en 1993 con 15 t.año-1 . En 1994, se constituyeron en la cuarta especie con 2.309 t.año ${ }^{-1}$. .Para 1998, se registraron 70 t.año $^{-1}$ (INPA, 1995, 1996, 1997, 
1999; Alvarado-Forero \& Gutiérrez-Bonilla, 2002). Lo cual permite un análisis comparado de los desembarcos pesqueros continentales a nivel nacional,

En los estudios pesqueros del río Sinú, de las 27 especies desembarcadas por los pescadores artesanales, y sobre un total para la cuenca de 1.800 t.año $^{-1}$, las tilapias representaron el $54 \%\left(234\right.$ t.año $\left.^{-1}\right)$, en la parte baja de la cuenca. En 1998, representaron el $24 \%$ sobre un total estimado de 2.512 t.año $^{-1}$ y en 2005 se mantuvo estable. Datos recientes sobre desembarcos pesqueros no existen. $\mathrm{Y} O$. niloticus ha tomado una gran ventaja sobre las poblaciones naturales y sobre todo en las Ciénagas de la Margen Izquierda (CMI) con 450 ha, pues Valderrama-Barco et al. (2002) registran que constituyó el $42,6 \%$ de las capturas en el año 2000 (predominando sobre el $P$. magdalenae) y el $13 \%$ en la Ciénaga Grande Lorica (CGL) con 38.000 ha, mientras que en los años anteriores constituía el $3,3 \%$ y el $2,2 \%$ de captura respectivamente (ValderramaBarco \& Ruiz, 1998). Para el año pesquero 2001-2002, Valderrama-Barco et al. (2002) registraron que la CGL aportó el $17,3 \%$, las CMI el $43,1 \%$ $\left(31,80\right.$ t.año $\left.^{-1}\right)$ y a nivel de la cuenca con el $14 \%\left(251,1\right.$ t.año $\left.{ }^{-1}\right)$ lo que significa una población desembarcada de aproximadamente 900.000 individuos. Económicamente, entre marzo de 2001 y febrero de 2002, O. niloticus aportó en las CMI 42,5 millones de pesos y en la CGL 450,8 millones de pesos.

La producción de O. niloticus en la cuenca hidrográfica del río Sinú $(251,1$ t.año ${ }^{-1}$ ) puede no ser comparable con algunos altos rendimientos en otras áreas de América Latina, pero el que se haya estimado como rendimiento poblacional estable el valor de 2.158 t.año $^{-1}$ (Gutiérrez-Bonilla, 2005), sí resulta comparable con lo establecido por Bonetto y Castello (1985) que listaron las principales especies introducidas en América Tropical y encontraron que todas han conllevado efectos negativos, estando entre ellas las tilapias. En Brasil, por ejemplo, contribuyen con el $30 \%$ en los reservorios DNOCS; en México son el $65-72 \%$ de las capturas, y entre estos están, el Lago Chapala, en donde son el $70 \%$ de $26 \mathrm{~kg} / \mathrm{ha} / \mathrm{año}{ }^{-1}$; en el reservorio Miguel Alemán, las capturas son de $40 \mathrm{~kg} / \mathrm{ha} / \mathrm{año}^{-1}$, y $90 \%$ de estas son tilapias; en República Dominicana entre el 70 y $90 \%$ de las pesquerías; en Cuba, la producción media anual de tilapias entre 1970 y 1974 fue de 14 t.año $^{-1}$, de 1975 a 1979 fue de 543 t.año $^{-1}$ y de 1980 a 1984 fue de 9.967 t.año $^{-1}$ (Díaz-Sarmiento \& Álvarez-León 1998), lo que significa el $93 \%$ del total de las capturas (10.659 t.año ${ }^{-1}$ ) (GutiérrezBonilla, 2005).

Todos los análisis permiten concluir que las poblaciones de O. niloticus, a escala nacional, se encuentran en buenas condiciones biológicopesqueras, plenamente establecidas y ensambladas. Este es el caso de la Ciénaga Grande de Santa Marta (CGSM), que ha sido invadida por el ingreso de los stocks procedentes de los ríos: Grande de La Magdalena $\left(256.000 \mathrm{~km}^{2}\right)$, Cauca $\left(63.300 \mathrm{~km}^{2}\right)$ y San Jorge $\left(14.800 \mathrm{~km}^{2}\right)$, estimándose que el número de individuos en las capturas en la CGSM, entre los años 2000-2001 y 2002, correspondieron a 18'600.096 x 10 ; $4,187.297 \times 10^{3}$ y $882.843 \times 10^{3}$ individuos respectivamente (INVEMAR, 2002). Establecida la O. niloticus, a escala nacional en las aguas continentales y con altas producciones pesqueras, se quiso saber en las ciénagas del norte de Colombia a qué se debía su éxito poblacional, su grado de adaptación y capacidad colonizadora de nuevos hábitats. Con este fin Narváez-Barandica et al. (2005) y Narváez-Barandica (2006), a través de análisis morfométricos y genéticos, concluyen que todo se debe a en gran parte a una interacción entre las capacidades ecológicas (entendida en la tolerancia a condiciones ambientales y poco exigentes en la alimentación), biológicas (entendida por el tipo de reproducción, cuidado parental y machos poligámicos) y genéticas (entendida en altos valores de variabilidad genética y fenotípica). Y establece, como lo han hecho otros estudios, que en Colombia al éxito de O. niloticus se le podría 
sumar: (1) los programas de repoblamiento adelantados por el Estado a través de sus estaciones piscícolas, que utilizaban las diferentes especies de tilapias, o una mezcla de las mismas (progenies) de las cepas de $O$. niloticus procedentes de diferentes países, y (2) la posibilidad de que la influencia del ambiente sobre sus poblaciones haya tenido como consecuencia la presencia de fenotipos específicos para cada ciénaga como un mecanismo de adaptación, puesto que las condiciones físicas, químicas y edáficas son muy diferentes en cada una ellas.

La tilapia roja (Oreochromis spp.), está ampliamente diseminada a nivel nacional a excepción del departamento del Guainía y de manera muy localizada en la cuenca del Amazonas. Y como tri-híbrido o tetra-híbrido reversado hormonal y genéticamente inestable, se encuentra distribuida por fugas y mal manejo de los cultivos, en los canales intermareales del Pacífico (Valverde-Pretelt \& Álvarez-León, 2002), lagunas estuarinas del Mar Caribe (Mercado-Silgado \& Álvarez-León, 2003), en donde también se engorda en cercos y en jaulas flotantes (Wedler, (1994a, 1994b). Los técnicos en sus informes y registros afirman: "contrariamente a lo esperado, sí hay reproducción del híbrido en agua salina” (VásquezArango \& Chaparro-Muñoz, 1997). Como ambiente artificial su mayor presencia ocurre en el Embalse de Betania (Huila), donde las mayores producciones en jaulas ascienden a 12.000 t (Gutiérrez-Bonilla, 2009).

En el Embalse de Betania (departamento del Huila) la producción de cíclidos introducidos (O. niloticus y Oreochromis spp.) aporta 678 t/año, a lo que debe sumarse la cifra ya relacionada de cultivo en jaulas (12.000 t), para un total de 12.678 t. La acuicultura en jaulas maneja aproximadamente 61,5 millones de individuos de $O$. niloticus, de Oreochromis spp. y de O. niloticus var. chitralada (Gutiérrez-Bonilla, 2009).

\section{MANIPULACIÓN GENÉTICA}

Especial mención merecen los impactos genéticos que, sobre las poblaciones nativas o naturalizadas de especies foráneas, pueden ocurrir por la introducción de híbridos, o nuevas especies, máxime cuando existan especies homólogas.

A Colombia se han introducido entre otros híbridos (Tabla 2): Oreochromis mossambicus - albina, tilapia roja singapur; Oreochromis spp., tilapia roja de la línea egipcia; Oreochromis spp., tilapia roja de la línea ghanesa; Oreochromis spp., tilapia roja de la línea tailandesa. $Y$ se produjeron entre otros híbridos: O. mossambicus x O. niloticus x O. aureus, Red Aurea, tilapia roja Israelí; O. mossambicus x 0 . urolepis - 0 . hornorum x 0 . niloticus $\times$ O. aureus; $O$. mossambicus $\times 0$. urolepis - $O$. hornorum - tilapia roja floridiana; $O$. mossambicus $\times 0$. niloticus - tilapia roja taiwanesa; $O$. mossambicus $\times$ O. urolepis - O. hornorum x O. niloticus, que se denominó la "Red Yumbo o tilapia roja colombiana"; O. niloticus x O. mossambicus, denominada Red Sterling, tilapia roja sterling o Swansea mutante; $O$. niloticus x $O$. aureus - tilapia roja, tilapia roja israelí. Este panorama de híbridos importados y los producidos con intereses comerciales, ampliamente diseminados por todo el país y utilizados en actividades de fomento y hasta de repoblación, hace imposible hablar de líneas genéticas puras. Finalmente, cuando en 2004 se importa la variedad tilapia tailandesa o chitralada, descendiente de líneas de $O$. niloticus de Egipto y Japón, se complejiza aún más el panorama de lo que pueda estar ocurriendo en la producción de híbridos para la acuicultura, pues con seguridad todos han pasado al medio natural (Gutiérrez-Bonilla, 2005).

Algunas investigaciones han intentado evaluar los impactos genéticos de los cíclidos y/o sus híbridos, pero el no tener información genética precisa 
sobre los pies parentales importados, ha generado imposibilidad para predecirlos. La introducción comercial al país de diferentes híbridos y cepas de Oreochromis spp. con características genéticas específicas, genera multiplicidad de arreglos entre las diferentes progenies en diversa cuencas, los cuales no se conocen. Alvarado-Forero y Gutiérrez-Bonilla (2002) capturaron en diversas cuencas continentales individuos con aletas dorsales y pectorales reducidas y ausencia de globo ocular, muestra de afectaciones genéticas, características que se repitieron en muestreos realizados entre 2003 y 2005 (Gutiérrez-Bonilla, 2005).

O. niloticus, en la cuenca del río Sinú, presentó un número modal cromosómico diploide $2 \mathrm{n}=44$; un número fundamental $\mathrm{F}=22$; 1 par de cromosomas metacéntricos, 14 pares de cromosomas submetacéntricos, 7 pares de cromosomas subtelocéntricos, para una fórmula cariotípica: $1 m+9 s m+5 s m+7 s t$., lo que concuerda con las investigaciones para la especie de Jalabert et al. (1971), Arai y Koike (1980), Majudmar y McAndrew (1986) y Yaseen (1993).

Sin embargo, es necesario anotar que otros autores han establecido un $2 \mathrm{n}$ $=40$. Bolaños et al. (1994) en un estudio citogenético de ejemplares de $O$. niloticus, que tenían del departamento del Cauca -aunque sin registrar su origen, presumiblemente para esa fecha eran del Valle del Cauca-, registran $2 n=44$, lo que denota el origen común de los pies parentales a nivel nacional.

Las actividades de repoblación con cíclidos y lo incorporado poblacionalmente a las cuencas hidrográficas nacionales, en seguimiento a las estadísticas aportadas por las autoridades ambientales, la autoridad pesquera nacional y las estaciones piscícolas a través de las actividades de fomento arrojan 16.000 millones de individuos, cifra que se corresponde con los aportes registrados en la pesquería.

En un inventario preliminar de las especies introducidas de cíclidos a Colombia, estas ascienden a 26 , siendo utilizadas para la acuicultura o en la acuariología (Tabla 1) y provienen de un amplio rango de la distribución mundial.

En 2000, la UICN a través del grupo de especialistas en especies invasivas estableció que O. mossambicus está entre las 100 más peligrosas especies invasoras (Lowe et al., 2002). Otras especies o variedades de tilapias (O. niloticus y Oreochromis spp.) en la "Conferencia sobre Especies Invasoras para Centroamérica y el Caribe", fueron reconocidas como invasoras (Hernández et al., 2002).

\section{TRASPLANTES}

El gran número de hibridaciones de que se tiene noticia en Florida (USA), fue producido por acuaristas o por la industria de peces ornamentales (Courtenay \& Robins, 1979), teniéndose 20 especies exóticas y 5 híbridas que habían establecido poblaciones reproductivas. Situación semejante ha ocurrido en el Lago de Nicaragua y en los embalses de Betania (departamento del Huila) y del Prado (departamento del Tolima) en Colombia, donde la tilapia (Oreochromis spp.) se ha convertido en la especie más abundante (McKaye, 1977; McKaye et al., 1995; AlvaradoForero, 1998) siendo objeto de una intensa actividad de pesca artesanal y acuacultura, que al 2009 representaba junto con O. niloticus y O. niloticus var. chitralada las especies objeto de cría y levante en los estanques y en los embalses. 
El hecho de que el Gobierno Nacional haya fomentado y mantenido un presupuesto más o menos creciente en el período 1984-1990, fue un índice alentador de lo que podía hacerse dentro de la actividad, sin olvidar que los principales productos de la acuicultura colombiana son de origen exótico (trucha y tilapia), o han sido trasplantados (camarón, cachamas) y que en 2000 la UICN, a través del grupo de especialistas en especies invasivas, reconoció a O. mossambicus entre las 100 más peligrosas especies invasoras (Lowe et al., 2002), y que otras especies variedades de tilapias en la "Conferencia sobre Especies Invasoras para Centroamérica y el Caribe", reconoció a otras especies de tilapias ( $O$. niloticus y Oreochromis spp.) como invasoras (Hernández et al., 2002).

La piscicultura en Colombia ha tenido un intenso trabajo genético, como puede verse en la Tabla 2, pues se han introducido padrotes de diversas partes del mundo y realizado diversos ensayos de cruces en los laboratorios de las fincas piscícolas, con el objeto de mejorar diversos aspectos de los peces, en la mayoría de los casos, presionados por el creciente y exigente comercio mundial, regional y nacional.

Recientemente, a la luz de la importancia nacional e internacional de la producción de tilapias en Colombia, Martínez (2008) evaluó el cultivo de la tilapia plateada (O. niloticus) y la tilapia roja (Oreochromis spp.) en diferentes sistemas intensivos de cultivo; Gitterle-Santamaría (2008), formuló a nombre de ACUANAL el Programa Nacional de Mejoramiento Genético de Tilapia Plateada; Salazar-Vallejo (2008), determinó la variabilidad genética de las poblaciones de tilapia roja mediante el uso de microsatélites; Bonilla (2008), presentó el modelo de simulación nacional de buenas prácticas de producción acuícola (BPPA) para la tilapia bajo el esquema GLOBALGAP; y Santamaría (2008), analizo la Normalización del Sector Acuícola a la luz del Compendio Acuícola ICONTECNOREXPORT.

\section{CONCLUSIONES}

La introducción de especies a Colombia se remonta al arribo de los españoles, pero se reinició en 1930 con el ingreso de las carpas (Cyprinidae) y las truchas y los salmones (Salmonidae). En 1959, comenzó la introducción de las tilapias (Cichlidae) con fines experimentales (Oreochromis mossambicus), pero accidental o voluntariamente esta especie y las posteriormente introducidas pasaron al medio natural. A la fecha se tiene registrada la introducción de 24 especies de cíclidos, 12 de estas presentes en ecosistemas dulceacuícolas y estuarinos, libres o confinados. La no realización de estudios sobre las especies nativas y la declinación de las poblaciones ícticas nativas objeto de aprovechamiento comercial, condujo a que en los últimos 15 años y como solución, se efectuaran repoblaciones con aproximadamente $16 \times 10^{6}$ individuos, de especies nativas trasplantadas y foráneas, sin prever nunca sus impactos negativos. A su vez, la producción acuícola está soportada a 2009 , en un $73,54 \%\left(57.286\right.$ t.año $\left.^{-1}\right)$ en especies foráneas, nativas trasplantadas y en híbridos, que han pasado al medio natural, sin que in situ se hayan determinado sus impactos.

Las reuniones de evaluación e identificación científica y técnica, han marcado las líneas de acción e investigación prioritarias, han permitido también la formulación de los Programas Nacionales y Regionales para la Acuicultura, y de paso identificar los proyectos prioritarios y la orientación de los fondos destinados a la investigación, el fomento y la 
comercialización. Las líneas de acción respecto a las introducciones y trasplantes, en cambio no han sido implementadas, haciéndose todo lo contrario.

En contravía a todo lo establecido en las investigaciones nacionales e internacionales, a 2009, el Estado intenta formular una reglamentación que ampliaría el espectro de utilización de las tilapias a nivel nacional, estableciendo una reglamentación de bioseguridad, pero los impactos son ya casi insolucionables. Estas acciones, simplemente darán vía libre a la utilización en todos los ecosistemas (abiertos o cerrados) de especies que solamente debieran ser utilizadas en estricto confinamiento.

A 2011, entre peces, moluscos y crustáceos introducidos el registro se acerca a las 170 especies, sin que se haya valorado su evolución poblacional o sus potenciales impactos negativos.

Nacionalmente aún estamos lejos de un verdadero diagnóstico y aclaración de los impactos derivados de las introducciones, trasplantes y acciones de repoblación, y ahora parecen imponerse las líneas de OVM, como la que tiene Cuba para tilapia, y a futuro los resultados derivados de las investigaciones sobre otras especies exóticas, tal como establece la FAO $(1981,2011)$ y COPESCAL (1986) que registran precisamente que es sobre especies exóticas sobre lo cual se hace el mayor número de investigaciones (S. trutta, C. carpio, M. salmoides, O. mossambicus, O. niloticus, O. mykiss y Gambusia affinis), con varios fines: resistencia al frío, mayor crecimiento y eficiencia de la alimentación, herencia estable, y resistencia a enfermedades.

\section{REFERENCIAS}

- Acero-Sánchez, A., y Hernández-Camacho, J. I. (1971). Apuntes sobre la carpa (Cyprinus carpio L. 1758), frente al desarrollo de la piscicultura en Colombia. Primer Seminario Nacional de Piscicultura. Manizales, Caldas. 17p.

- Allan, J. D., y Flecker, A. S. (1993). Biodiversity conservation in running waters: identifying the major factors that affect destruction of riverine species and ecosystems. BioScience, 43, 497-502.

- Alvarado-Forero, H. (1998). Plan de ordenamiento pesquero del Embalse de Betania (Huila). Santa Fe de Bogotá, D.C.: INPA. Inf. Técnico. 38p.

- Alvarado-Forero, H., y Gutiérrez-Bonilla, F. de P. (1999). Especies hidrobiológicas continentales introducidas y trasplantadas y su distribución en Colombia. Santa Fe de Bogotá D.C., Colombia: Ministerio del Medio Ambiente / Instituto de Investigaciones de los Recursos Biológicos Alexander von Humboldt. Inf. Técnico. $134 p$.

- _ (2002). Especies hidrobiológicas continentales introducidas y trasplantadas y su distribución en Colombia. Santa Fe de Bogotá D.C., Colombia: Ministerio del Medio Ambiente / Instituto de Investigaciones de los Recursos Biológicos Alexander von Humboldt. 181p.

- Álvarez-León, R., y Rodríguez-Forero, A. (2000). La acuicultura en Colombia: estado actual y perspectivas. INFOPESCA Internacional, 6, 40-47.

- Álvarez-León, R., Gutiérrez-Bonilla, F. de P., y Rodríguez-Forero, A. (2002). La introducción y trasplante de peces dulceacuícolas en Colombia: impactos ecológicos, económicos y legales. En Mojica-Corso, J. I., Castellanos-Castillo, C., Usma-Oviedo, J. S., 
y Álvarez-León, R. (Eds.), El libro rojo de los peces dulceacuícolas de Colombia (pp. 55-62 + 270-274). La Serie de Libros Rojos de Especies Amenazadas de Colombia. Santa Fe de Bogotá D.C., Colombia: ICN-UNC / IIBAVH / MINAMBIENTE / Cl-Colombia. 285 p.

- Arai, R. y, Koike, A. (1980). A karyotype study on two species of freshwater fishes transplanted into Japan. Bull. Nat. Sci. Mus., Ser. 6(4), 275-286.

- Baptiste, M., Castaño, N., Cárdenas, D., Gutiérrez, F. de P., Gil, D., y Lasso-Alcalá, C. A. (2010). Análisis de riesgo y propuesta de categorización de especies introducidas para Colombia. Bogotá D.C.: Instituto Alexander von Humboldt. 220p.

- Beltrán-Turriago, C. S., Villaneda, A. A., Díaz, F. J., Carrillo, M., y Salazar-Ariza, G. (s.f.). Paquetes productivos en acuicultura: cachama, trucha y tilapia. Bogotá D.C.: INPA. Inf. Técnico. 17p.

- Bolaños, L., Camayo, C., y Penna, L. (1994). Estudio citogenético de las especies ícticas: Piaractus brachypomus, Prochilodus reticulatus, Oreochromis niloticus, Oreochromis mossambicus y Oreochromis spp. Tesis Profesional, Fac. de Biología, Univ. del Cauca.

- Bonetto, A., y Castello, H. (1985). Pesca y piscicultura en aguas continentales de América Latina. OEA. Biol. Ser. Monogr., 31, 1100.

- Bonilla, S. P. (2008). Presentación del modelo simulado nacional de buenas prácticas de producción acuícola (BPPA) en el producto tilapia bajo el esquema GLOBALGAP. Encuentro Nacional de Productores de Tilapia en torno a las Buenas Prácticas de Producción Acuícola (BPPA). CENIACUA / SENA. Neiva (Huila), Mayo 15 y 16 de 2008.

- Burbano, C., y Usaquén, W. (2003). Caracterización genética de cinco especies ícticas del río Sinú (Caquetaia kraussi, Brycon moorei, Prochilodus magdalenae, Pimelodus clarias, Sorubim lima). Proyecto hidroeléctrico URRÁ. Bogotá D.C: Universidad Nacional de Colombia, Departamento de Biología. 120p.

- CEP-UDC. (1973-1983). Ensayos de cultivo con Tilapia rendalli y Oreochromis nilotica, para evaluar su comportamiento frente a especies nativas, trasplantadas e introducidas. Centro Piscícola Experimental, Universidad de Caldas. Informes Técnicos 1-3. Manizales, Colombia. 165p.

- Contreras, B. S. (2002). Base de datos del proyecto AE002 Especies de peces introducidas en aguas continentales de México. Catálogo y manuscrito. Proyecto en seguimiento SNIBCONABIO. México D.F.

- Contreras, S., y Escalante, M. A. (1984). Distribution and known impacts of exotic fishes in Mexico. En Courtenay, W. R. (Ed.), Management of exotic Fishes (pp. 102-130). Baltimore (MD), USA: John Hopkins University Press.

- Contreras-Balderas, S. (1999). Annotated checklist of introduced invasive fishes in Mexico, with examples of some recent introductions. En Claudi, R., y Leach, J. H. (Eds.), Nonindigenous freshwater fishes-vectors, biology, and impacts (chapter 2, pp. 33-54). Washington, USA: Lewis Publ.

- COPESCAL. (1986). Introducción de especies ícticas y conservación de los recursos genéticos de América Latina y el Caribe. Documento Ocasional, 3, 1-12. FAO / COPESCAL.

- Courtenay, W. R. Jr., y Robins, C. R. (1979). Exotic aquatic organisms in Florida with emphasis on fishes: A review and recommendations. Transactions of The American Fisheries Society, 102, 1-12. 
- Dahl, G. (1958). Los peces del río Sinú. Informe preliminar. Montería (Córdoba), Colombia: Pub. Secretaría de Agricultura y Ganadería de Córdoba, Impr. Deptal. 47p.

- De longh, H. H., y Van Zon, J. C. J. (1993). Assessment of impact of the introduction of exotic fish species in northeast Thailand. Aquaculture and Fisheries Management, 24(3), 279-289.

- Devlin, R. H. L., Sundtrom, F., Jhonson, J. I., Fleming, L. A., Hayes, K. R., Ojwang, W. O., Bambaradeniya, C., y Zakara-Ismail, M. (2007). Biological invasions in marine ecosystems. Ecological management, and geographic perspectives. En Rilov, G., y Crooks, J. A. (Eds.), 2007. Ecological management, and geographic perspectives. Ecological Studies, 204, 3, 151-187.

- Díaz-Sarmiento, J. A., y Álvarez-León, R. (1998). Fish Biodiversity Conservation in Colombia. En Harvey, B., Ross, C., Greer, D., y Carolsfeld, J. (Eds.), Action Before Extinction (pp. 215-223). International-Development-Research-Centre-IRDC, OttawaCanada; International Development Research Centre IRDC, Ottawa, Canada 202-505-Fisgard-St World-Fisheries-Trust 1998. 259p.

- Erazo-Killer, A. (1989). Introduction of exotic species for fish culture at the Llanos Orientales Region. Mem. of the Workshop on Introduction of Hydrobiologic Species to Aquaculture. pp. 43-46.

- FAO. (1981). Situación actual de la acuacultura en América Latina. $2^{a}$ Reunión Com. Pesca Continental para América Latina (COPESCAL). Santo Domingo (Rep. Dominicana), dic. 2-4. 14 p.

- (2011). Directrices técnicas para la certificación en acuicultura. Versión aprobado por los miembros el comité de pesca (COFI) en su vigésimo novena sesión celebrada en Roma, Italia del 31 de enero al 4 de febrero del 2011. Roma (Italia). 31p.

- Font, W. F. (2003). The global spread of parasites: What do Hawaiian streams tell us? BioScience, 53(11), 1061-1067.

- Garzón, F. (1990). La piscicultura de fomento en Colombia: análisis de la situación actual. Bogotá D.E., Colombia: Fondo DRI. 64p.

- GISP. (2005). Sudamérica invadida. El creciente peligro de las especies exóticas invasoras. El Programa Mundial sobre Especies Invasoras. 80p.

- Gitterle-Santamaría, T. (2008). Programa nacional de mejoramiento genético de tilapia nilótica. Encuentro Nacional de Productores de Tilapia en torno a las Buenas Prácticas de Producción Acuícola (BPPA). CENIACUA / SENA. Neiva (Huila), Mayo 15 y 16 de 2008.

- Gutiérrez-Bonilla, F. de P. (2001). La introducción de especies como un fenómeno global y las especies hidrobiológicas continentales introducidas $y / 0$ trasplantadas en Colombia. Asociación Luna Roja - Rev. Medio Ambiente y Desarrollo Sostenible, 5(14), 3-32.

- _ (2005). Distribución de las especies hidrobiológicas continentales introducidas y/o traslocadas en Colombia. Caso de Estudio: Biología y ecología de Oreochromis niloticus en la Cuenca Hidrográfica del Río Sinú. Tesis Doctoral., Departament de Biología Animal (Vertebrats), Facultat de Biología, Universitat de Barcelona. 350p.

- _ (2009). La actividad pesquera en el embalse de Betania (Huila). Ajuste al plan de ordenamiento pesquero y acuícola del embalse de Betania. Bogotá D.C., Colombia: Fac. de Biol. Marina, Univ. de Bogotá Jorge Tadeo Lozano. 60p.

- _ (2010). Los recursos hidrobiológicos y pesqueros continentales en Colombia. Bogotá D.C., Colombia: Instituto de Investigación de Recursos Biológicos Alexander von Humboldt. $118 p$. 
- Hall, S. R., y Mills, E. L. (2000). Exotic species in large lakes of the world. Aquatic Ecosystem Health and Management, 3, 105-135.

- Harrison, I. J., y Stiassny, M. J. (1999). The Quiet Crisis: A Preliminary Listing of the Freshwater Fishes of the World that Are Extinct or 'Missing in Action, pp. 271-331. En MacPhee (Ed.), Extinctions in Near Time. New York, USA: Kluwer Academic/Plenum Publishers.

- Hernández, G., Lahmann, E., y Pérez-Gil, R. (2002). Invasores en Mesoamérica y el Caribe. San José, Costa Rica: IUCN. 110p.

- Hopkins, C. C. E. (2001). Actual and potential effects of introduced marine organisms in Norwegian waters, including $S$ Gopal, B.1987. Water hyacinth Aquatic Plant. Studies 1. Amsterdam, The Netherlands: Elsevier Science. 471p.

- ICA. (2008). Evaluación de recursos pesqueros colombianos. Bogotá D.C., Colombia: Instituto Colombiano Agropecuario, Subgerencia de Pesca y Acuicultura. 131p.

- Infante, O. (1985). Aspectos bioecológicos de la tilapia Sarotherodon mossambicus (Peters 1852, Teleostei, Perciformes, Cichlidae) en el lago de Valencia, Venezuela. Acta Científica Venezolana, 36, 68-76.

- INPA. (1995). Boletín de Estadísticas Pesqueras, 1994. Santafé de Bogotá D.C., Colombia: Instituto Nacional de Pesca y Acuicultura. s.p. (1996). Boletín de Estadísticas Pesqueras, 1995. Santafé de Bogotá D.C., Colombia: Instituto Nacional de Pesca y Acuicultura. $107 \mathrm{p}$

(1997). Boletín de Estadísticas Pesqueras, 1996. Santafé de Bogotá D.C., Colombia: Instituto Nacional de Pesca y Acuicultura. 110p.

- _ (1999). Boletín de Estadísticas Pesqueras, 1997 y 1998. Santafé de Bogotá D.C., Colombia: Instituto Nacional de Pesca y Acuicultura. 114p.

- INVEMAR. (2002). Monitoreo de las condiciones ambientales y los cambios estructurales y funcionales de las comunidades vegetales y de los recursos pesqueros durante la rehabilitación de la CGSM: Un enfoque adaptativo. Inf. Técnico Final 19992002. Santa Marta, Colombia: Instituto de Investigaciones Marinas y Costeras "José Benito Vives de Andreis". 232p.

- IUCN. (2008). La biodiversidad de agua dulce. Un recurso Escondido y amenazado. Unión Internacional para la Conservación de la Naturaleza. Red List. 2p.

- Jalabert, B., Kammacher, P., y Lessent, P. (1971). Determinisme du sexe chez les hybrides du gendre Tilapia. Ann. Biol. Anim. Bioch. Bioph., 11, 155-165.

- Kingston, N., y Waldren, S. (2003). The plant communities and environmental gradients of Pitcarian island: The significance of invasive species and the need for conservation management. Annals of Botany, 92(1), 31-34.

- Lachner, E. A., Robins, C. R., y Courtenay, W. R. Jr. (1970). Exotic fishes and other aquatic organisms introduced into North America. Smithsonian Contrib. Zool., 59, 1-29.

- Lassuy, D. R. (2002). Introduced species as a factor in extinction and endangerment of native fish species. Workshop: Management, Implications and Co-occurring Native and Introduced Fishes Proceedings, Portland (Oregon), USA. pp. 2728.

- Lee, C, E., Remfert, J, L., y Gelembiuk, G. (2003). Evolution of physiological tolerance and perfomance during freshwater invasions. Integrative \& Comparativy Biology, 43(3), 439-49.

- López-Macías, F. J., Buitrago-López, M., y Reyes-González, J. F. (2007). Viabilidad de una truchifactoría como alternativa de 
producción sostenible en la zona del Páramo de Letras, Caldas y Tolima, Colombia. Veterinaria y Zootecnia, 1(2), 30-42.

- Lovshin, L. (1980). Situación del cultivo de Colossoma spp. en Sudamérica. Rev. Lat.-Amer. de Acuic., 5, 27-32.

- Lowe, S., Browse, M., y Boudelas, S. (2002). 100 of the world's worst invasive alien species. A selection from the Invasive Species Database. Auckland, New Zealand: Invasive Species Specialist Group. 11p.

- MADR / CCl. (2010). Pesca y Acuicultura en Colombia 2009. Informe Técnico Regional Cuencas del Magdalena, Sinú y Atrato. Bogotá D.C., Colombia: Ministerio de Agricultura y Desarrollo Rural / Corporación Colombia Internacional. 76 p.

- Mago-Leccia, F. (1978). Los peces de agua dulce de Venezuela. Cuadernos Lagoven. Caracas: Editorial Cromotip. 35p.

- Majudmar, K. C., y McAndrew, B. J. (1986). Relative DNA content of somatic nuclei and chromosomal studies in three genera: Tilapia, Sarotherodon, and Oreochromis of the tribe Tilapiini (Pisces: Cichlidae). Genetic, 68(3), 175-188.

- Martínez, X. (2008). Evaluación del cultivo de la tilapia del nilo (Oreochromis niloticus) y la tilapia roja (Oreochromis spp.) en diferentes sistemas intensivos de cultivo en Colombia. Encuentro Nacional de Productores de Tilapia en torno a las Buenas Prácticas de Producción Acuícola (BPPA). CENIACUA / SENA. Neiva (Huila), Mayo 15 y 16 de 2008.

- Martínez-Espinosa, M. (1984). El cultivo de las especies del género Colossoma en América Latina. Consultoría FAO-RLAC/84/41PES-5. Santiago, Chile. 46p.

- McKaye, K. R. (1977). Competition for breeding sites between the cichlid fishes of Lake Jiloá, Nicaragua. Ecology, 58, 291-302.

- McKaye, K. R., Ryan, J. D., Sattuffer, J. R. Jr., López, L. J., Vega, G. I., y van der Berghe, E. P. (1995). African tilapia in Lake Nicaragua. BioScience, 45, 406-411.

- McNeely, J. A., Mooney, H. A., Neville, L. E., Schei, P., y Waage, J. K. (Eds.). (2001). A global strategy on invasive alien species. Global Invasive Special Programme-IUCN. Gland ( Switzerland), $60 \mathrm{p}$.

- Mercado-Silgado, J. E., y Álvarez-León, R. (2003). Piscicultura en Colombia: Experiencias en la zona costera del Caribe. INFOPESCA Internal., 13, 24-30.

- Moyle, P. B., y Leydy, R. L. (1992). Loss of biodiversity in aquatic ecosystems: evidence from fish faunas. En Fiedler, P. I., y Jain, S. K. (Eds.), Conservation biology: the theory and practice of nature conservation, preservation and management (pp. 127127). New York, USA: Chapman y Hall.

- Narváez-Barandica, J. C. (2006). Evaluación de la estructura genética y morfométrica de las poblaciones de tilapia (Pisces: Cichlidae: Oreochromis) en algunas ciénagas del norte de Colombia.

Tesis M.Sc., Fac. de Ciencias, Univ. Nacional de Colombia.

- Narváez-Barandica, J. C., Acero-Pizarro, A., y Blanco-Racedo, J. (2005). Variación morfométrica en poblaciones naturalizadas y domesticadas de la tilapia del Nilo, Oreochromis niloticus (Teleostei: Cichlidae) en el norte de Colombia. Rev. Acad. Colomb. Cienc., 29(112), 383-394.

- Otero, R. J. (1989). Introduction of exotic species for fish culture at the Llanos Orientales region. En Mem. of the Workshop on Introduction of Hydrobiologic Species to Aquaculture (pp. 43-46).

- Paredes, L. (1995). Determinación del impacto de la tilapia roja (Oreochromis spp.) sobre un policultivo de ocho especies nativas del Bajo Magdalena y una exótica (Oreochromis niloticus). Inf. Técnico. Bogotá D.C.: INPA. 
- Pérez, J. E., Graziani, C. A., y Nirchio, M. (1997). ¿Hasta cuándo los exóticos? Acta Científica Venezolana, 48(3), 127-129.

- Popma, T., y Lozano-Díaz, H. (1977a). Declaración de efecto ambiental sobre el cultivo de Tilapia rendalli en los sistemas magdalénico y caucano. Proyecto INDERENA-AID para el Desarrollo de la Acuicultura Continental en Colombia IA/514/T/078. Bogotá D.E., Colombia. pp. 1-17.

- _ (1977b). Declaración de efecto ambiental sobre el uso del pez Cichla ocellaris. Proyecto INDERENA-AID para el Desarrollo de la Acuicultura Continental en Colombia IA/514/T/078. Bogotá D.E., Colombia. pp. 1-10.

- _ (1977c). Declaración de efecto ambiental para la introducción al país y cultivo de Sarotherodon niloticus. Proyecto INDERENA-AID para el Desarrollo de la Acuicultura Continental en Colombia I-A/514/T/078. Bogotá D.E., Colombia. pp. 1-4.

- Popma, T., y Villanada-Jiménez, A. (1977). Sustentación de la introducción de Sarotherodon niloticus (tilapia nilótica) en Colombia. Proyecto INDERENA-AID para el Desarrollo de la Acuicultura Continental en Colombia I-A/514/T/078. Bogotá D.E., Colombia. pp. 1-4.

- Ramos-Henao, A. (1972). Fundamentos de piscicultura agrícola. Manizales, Caldas: Univ. de Caldas, Fac. de Veterinaria y Zootecnia, Sec. de Piscicultura / Comité Departamental de Cafeteros de Caldas. 52p.

- (1979). Fundamentos de piscicultura agrícola. Manizales, Caldas: Univ. de Caldas, Fac. de Veterinaria y Zootecnia, Sec. de Piscicultura / Comité Departamental de Cafeteros de Caldas. 56p.

- Rilov, G., y Crooks, J. A. (2007). Biological invasions in marine ecosystems. Ecological management, and geographic perspectives. Ecological studies 204. Edit. Springer. Oregon State University. (USA). 641p.

- Robles, C. A. (2008). Nueva institucionalidad para el sector de la pesca y la acuicultura. Rev. Colomb. Cienc. Pecua., 21(3), 461462.

- Rodríguez-Gómez, H. (1984). Peces exóticos introducidos y establecidos en aguas colombianas. Bogotá D.E., Colombia: INDERENA. 36p.

- Rodríguez-Guerrero, D., y Phelps, R. (1982). Determinación del efecto de la mojarra africana tilapia nilótica sobre una población de 13 especies de peces nativos del área del Bajo Magdalena. INDERENA. Rev. Divulg. Pesq., 22(1), 1-26.

- Royero-León, R., y Lasso-Alcalá, C. A. (1992). Distribución geográfica actual de la mojarra de río, Caquetaia kraussii, (Steindachner, 1878) (Perciformes, Cichlidae) en Venezuela: un ejemplo del problema de la introducción de especies. Memoria Sociedad Ciencias Naturales La Salle, 52(138), 163-180.

- Ruiz, G. (1997). The aliens among US. Chesapeake Bay. Program SERC. Internet. Aliens. USA. pp. 1-3.

- Salazar-Ariza, G. (1999). Situación de la acuicultura rural a pequeña escala en Colombia: Importancia, perspectivas $y$ estrategias de desarrollo. Red de Acuicultura Rural a Pequeña Escala. Taller ARPE, FAO-UCT, Nov. 9-12.

- Salazar-Vallejo, M. (2008). Determinación de la variabilidad genética de las poblaciones de tilapia roja mediante el uso de microsatélites. Encuentro Nacional de Productores de Tilapia en torno a las Buenas Prácticas de Producción Acuícola (BPPA). CENIACUA / SENA. Neiva (Huila), Mayo 15 y 16 de 2008.

- Santamaría, L. D. (2008). Normalización Sector AcuícolaLanzamiento Compendio Acuícola ICONTEC-NOREXPORT. Encuentro Nacional de Productores de Tilapia en torno a las 
Buenas Prácticas de Producción Acuícola (BPPA). CENIACUA / SENA. Neiva (Huila), Mayo 15 y 16 de 2008.

- Señaris, J. C., y Lasso-Alcalá, C. A. (1993). Ecología alimentaria y reproductiva de la mojarra de río, Caquetaia kraussii (Steindachner 1878) (Pisces: Cichlidae), en los Llanos inundables del Edo. Apure, Venezuela. Publicaciones Asociación Amigos de Doñana, 2, 1-58.

- Shelton, W. L., y Smitherman, R. O. (1984). Exotics fishes in warms water aquaculture. En Courtenay, W. R. Jr., y Satuffer, J. R. Jr. (Eds.), Distribution, Biology and Management of Exotics Fishes (pp. 262-301). Baltimore, USA: Johns Hopkins University Press.

- Siguan, M. (2003). Pathways of biological invasions of marine plants. En Ruiz, G. M., y Carlton, J. T. (Eds.), Invasive species: Vectors and management strategies (pp. 183-227). Washington, USA: Island Press. 518p.

- Solórzano, E., Marcano-Chirguita, C. W., Quijada, A., y Campo, M. (2001). Impacto ecosistémico de las tilapias introducidas en Venezuela. En Ojasti, J., González-Jiménez, E., SzeplakiOtahola, E., y García-Román, L. B. (Eds.), Informe sobre las especies exóticas en Venezuela (pp. 194-199). Caracas: Ministerio del Ambiente y de los Recursos Naturales, Editorial Tipodin.

- Valderrama-Barco, M., y Ruiz, O. (1998). Monitoreo pesquero del Medio y Bajo Sinú. Evaluación de la captura y esfuerzo y determinación de información biológico pesquera de las principales especies ícticas en las áreas de Lorica, Betancí y Tierralta. Inf. Final. Convenio INPA / URRÁ S.A. ESP. Montería, Colombia. 120p.

- Valderrama-Barco, M., Vejarano, S., Solano, D., Álvarez, L., Núñez, C., Jarava, E., y Pescadores del Sinú. (2002). Monitoreo y estadística pesquera de la Cuenca del río Sinú con participación comunitaria. Inf. Final. Convenio INPA / URRÁ S. A. Montería, Colombia. 105p.

- Valverde-Pretelt, J., y Álvarez-León, R. (2002). Piscicultura en Colombia: Experiencias en la zona costera del Pacífico. INFOPESCA Internal., 12, 20-27.

- Vásquez-Arango, J. G., y Chaparro-Muñoz, N. (1997). Aprovechamiento de productos agrícolas para el cultivo de la tilapia roja (Oreochromis spp.) en jaulas flotantes, transfiriendo tecnología a la comunidad, Golfo de Urabá, Caribe colombiano. En IV Simp. Colomb. de Ictiología (p. 76). ACICTIOS / INVEMAR UDM / INPA. Santa Marta, Agosto 7-10 de 1997. Resúmenes de Conferencias y Exposiciones. 103p.

- Wedler, E. (1994a). Cultivo de la tilapia roja en lagunas costeras bajo condiciones de salinidad fluctuante. Experimentos de engorde intensivo en jaulas en la Ciénaga Grande de Santa Marta, Colombia. Santa Marta, Colombia: Univ. del Magdalena. $105 p$.

- (1994b). El cultivo en jaulas de la tilapia roja en una laguna costera bajo condiciones de salinidad fluctuante. En Memorias VII Congreso Latinoamericano de Acuicultura. Santafé de Bogotá D.C., Colombia.

- Welcomme, R. L. (1981). Register of international transfers of inland fish species. FAO Fish. Tec. Pap., 213. Rome, Italy. 120p.

- (1988). International introductions of island aquatic species. FAO Fish. Tec. Pap., 294, 1-318.

- Yaseen, A. E. (1993). Chromosomes studies of Nile Tilapia nilotica in upper Egypt (Teleostei, Cichlidae). J. Egypt. Gen. Soc. Zool., 11(C), 79-88. 
1. Universidad de Bogotá Jorge Tadeo Lozano. Bogotá D.C., Colombia.

2. Fundaciones Maguaré y Verdes Horizontes. Manizales, Caldas, Colombia. 
Tabla 1. Cíclidos (Pisces: Perciformes: Cichlidae) exóticos presentes en Colombia

\begin{tabular}{|c|c|c|c|c|c|}
\hline $\begin{array}{c}\text { ESPECIE / NOMBRE } \\
\text { VERNACULAR }\end{array}$ & DESDE & AÑO & $\begin{array}{c}\text { ESTABLECIDO } \\
\text { (DEPARTAMENTO) }\end{array}$ & PROPÓSITO & ANOTACIÓN \\
\hline $\begin{array}{l}\text { 1. Amatitlania } \\
\text { nigrofasciata } \\
\text { Pez convicto, cíclido } \\
\text { zebra }\end{array}$ & CEN & $\begin{array}{l}1990 \\
1994\end{array}$ & $\begin{array}{l}2 \text { (Antioquia, } \\
\text { Cundinamarca, } \\
\text { Caldas) }\end{array}$ & 4 & 7,15 \\
\hline $\begin{array}{l}\text { 2. Amphilophus } \\
\text { macracanthus }\end{array}$ & CEN & - & $\begin{array}{c}2 \text { (Cundinamarca, } \\
\text { Caldas) }\end{array}$ & 4 & 7 \\
\hline $\begin{array}{l}\text { 3. Cyrtocara } \\
\text { compressiceps } \\
\text { Mordedor de ojos de } \\
\text { Malawi }\end{array}$ & AFC & $\begin{array}{l}1990 \\
1994\end{array}$ & $\begin{array}{l}2 \text { (Antioquia, } \\
\text { Cundinamarca) }\end{array}$ & 4 & 7 \\
\hline $\begin{array}{l}\text { 4. Hemichomis } \\
\text { bimaculatus } \\
\text { Cíclido encarnado, } \\
\text { cíclido joya, jewlfish }\end{array}$ & AFC & $\begin{array}{l}1990 \\
1994\end{array}$ & $\begin{array}{l}2 \text { (Antioquia, } \\
\text { Cundinamarca) }\end{array}$ & 4 & 7 \\
\hline $\begin{array}{l}\text { 5. Heros severus } \\
\text { Falso escalar }\end{array}$ & AMA & - & $\begin{array}{c}2 \text { (Cundinamarca, } \\
\text { Caldas) }\end{array}$ & 4 & 7 \\
\hline $\begin{array}{l}\text { 6. Etroplus maculatus } \\
\text { Cíclido naranja, cíclido } \\
\text { punteado, naranja } \\
\text { cromado }\end{array}$ & $\overline{A F C}$ & $\begin{array}{l}1990 \\
1994\end{array}$ & $\begin{array}{l}2 \text { (Antioquia, Bolívar, } \\
\text { Cundinamarca) }\end{array}$ & 4 & 7 \\
\hline $\begin{array}{l}\text { 7. Maylandia zebra } \\
\text { Cíclido azul de } \\
\text { Nyassa, cíclido cebra, } \\
\text { cíclido azul del Lago } \\
\text { Malawi, Zebra mbuna }\end{array}$ & AFC & $\begin{array}{l}1990 \\
1994\end{array}$ & $\begin{array}{l}2 \text { (Antioquia, } \\
\text { Cundinamarca, } \\
\text { Caldas) }\end{array}$ & 4 & 7,15 \\
\hline $\begin{array}{l}\text { 8. Melanochromis } \\
\text { auratus } \\
\text { Auratus, cíclido dorado } \\
\text { del Lago Malawi }\end{array}$ & $\overline{A F C}$ & $\begin{array}{l}1990 \\
1994\end{array}$ & $\begin{array}{l}2 \text { (Antioquia, } \\
\text { Cundinamarca) }\end{array}$ & 4 & 7 \\
\hline $\begin{array}{l}\text { 9. Melanochromis } \\
\text { johannii } \\
\text { Bluegray mbunai }\end{array}$ & AFC & - & 2 (Caldas) & 4 & 7,15 \\
\hline $\begin{array}{l}\text { 10. Neolamprologus } \\
\text { brichardi } \\
\text { Cíclido limón }\end{array}$ & AFC & - & 2 (Caldas) & 4 & 7,15 \\
\hline $\begin{array}{l}\text { 11. Neolanochromis } \\
\text { brichardi }\end{array}$ & AFC & $\begin{array}{l}1990 \\
1994\end{array}$ & $\begin{array}{c}2 \text { (Antioquia, } \\
\text { Cundinamarca) }\end{array}$ & 4 & 7 \\
\hline $\begin{array}{l}\text { 12. Oreochromis } \\
\text { (Sarotherodon) aureus } \\
\text { Tilapia aurea, tilapia } \\
\text { azul }\end{array}$ & PAN & 1989 & $\begin{array}{l}2 \text { (Bolívar, Caldas, } \\
\text { Valle, Magdalena) }\end{array}$ & 3,6 & 11 \\
\hline $\begin{array}{l}\text { 13. Oreochromis (S.) } \\
\text { hornorum } \\
\text { Tilapia hornorum }\end{array}$ & PAN & 1985 & $\begin{array}{l}2 \text { (Cuencas del } \\
\text { Magdalena, } \\
\text { Cauca) }\end{array}$ & 3,6 & 7 \\
\hline $\begin{array}{l}\text { 14. Oreochromis (S.) } \\
\text { mossambicus } \\
\text { Mozámbica, tilapia } \\
\text { negra }\end{array}$ & $\begin{array}{l}\text { USA, } \\
\text { JAM, } \\
\text { MEX }\end{array}$ & $\begin{array}{l}1953 \\
1959 \\
1959\end{array}$ & $\begin{array}{c}2 \text { (Cuencas } \\
\text { Magdalena, } \\
\text { Cauca, Vertiente } \\
\text { Pacífica) }\end{array}$ & 3,6 & 13 \\
\hline $\begin{array}{l}\text { 15. Oreochromis (S.) } \\
\text { mossambicus, albina }\end{array}$ & USA & 1981 & $\begin{array}{c}1 \text { (Cuencas } \\
\text { Magdalena, } \\
\text { Cauca) }\end{array}$ & 3 & 7 \\
\hline $\begin{array}{l}\text { 16. Oreochromis (S.) } \\
\text { niloticus niloticus } \\
\text { Nilótica, tilapia } \\
\text { plateada }\end{array}$ & PAN & 1979 & $\begin{array}{c}\text { 1,2 (Cuencas } \\
\text { Magdalena, Cauca, } \\
\text { Orinoco, Amazonas, } \\
\text { Vertientes del } \\
\text { Pacífico. Caribe) }\end{array}$ & 3,6 & 7,8 \\
\hline $\begin{array}{l}\text { 17. Oreochromis. (S.) } \\
\text { niloticus var. } \\
\text { chitralada, Chitralada }\end{array}$ & $\begin{array}{l}\text { BRA, } \\
\text { TAl }\end{array}$ & 2002 & $\begin{array}{l}2 \text { (Cuencas del } \\
\text { Magdalena, Meta) }\end{array}$ & 3 & 7 \\
\hline $\begin{array}{l}\text { 18. Oreochromis (S.) } \\
\text { urolepis hornorum } \\
\text { Tilapia hornorum }\end{array}$ & BRA & 1986 & $\begin{array}{l}2 \text { (Bolívar, Caldas, } \\
\text { Valle, Magdalena) }\end{array}$ & 3 & 11 \\
\hline $\begin{array}{l}\text { 19. Oreochromis spp. } \\
\text { Tilapia roja, mojarra } \\
\text { roja, trihibrido, } \\
\text { tetrahíbrido }\end{array}$ & & 1981 & $\begin{array}{l}2 \text { (Todo el país, } \\
\text { excepto el } \\
\text { departamento de } \\
\text { Guainía) }\end{array}$ & & \\
\hline $\begin{array}{l}\text { 20. Pelvicachromis } \\
\text { pulcher } \\
\text { Raimbow krib }\end{array}$ & $\overline{A F C}$ & - & 2 (Caldas) & 4 & 7,15 \\
\hline $\begin{array}{l}\text { 21. Pseudotropheus } \\
\text { elongatus } \\
\text { Elongatus, elongate } \\
\text { mbuna, mbuna } \\
\text { alargada. }\end{array}$ & AFC & $\begin{array}{l}1990 \\
1994\end{array}$ & $\begin{array}{l}2 \text { (Antioquia, } \\
\text { Cundinamarca) }\end{array}$ & 4 & 7 \\
\hline $\begin{array}{l}\text { 22. Rocio octofasciata } \\
\text { Jack dempsey }\end{array}$ & CEN & - & 2 (Caldas) & 4 & 7,15 \\
\hline $\begin{array}{l}\text { 23. Tilapia rendalli } \\
\text { Rendali, tilapia } \\
\text { hervibora }\end{array}$ & BRA & 1960 & $\begin{array}{c}2 \text { (Cuencas } \\
\text { Magdalena, } \\
\text { Cauca, Eje Cafetero, } \\
\text { Catatumbo, } \\
\text { Vertientes Pacífica, } \\
\text { Caribe) }\end{array}$ & 3,6 & 7,13 \\
\hline $\begin{array}{l}\text { 24. Thorichthys meeki } \\
\text { Boca de fuego }\end{array}$ & CEN & - & 2 (Caldas, Valle) & 4 & $7,11,15$ \\
\hline $\begin{array}{l}\text { 25. Tropheops gracilior } \\
\text { Amarillo }\end{array}$ & AFC & $\begin{array}{l}1990 \\
1994\end{array}$ & 2 (Caldas) & 4 & 7,15 \\
\hline $\begin{array}{l}\text { 26. Tropheops } \\
\text { tropheops } \\
\text { Tropheus dorado }\end{array}$ & $\overline{\mathrm{AFC}}$ & $\begin{array}{l}1990 \\
1994\end{array}$ & $\begin{array}{l}2 \text { (Antioquia, } \\
\text { Cundinamarca, } \\
\text { Caldas) }\end{array}$ & 4 & 7,15 \\
\hline
\end{tabular}


Establecidas en: (1) ambientes naturales, (2) ambientes artificiales; propósito: (3) acuicultura, (4) ornamental, (5) control de mosquitos; observaciones: (6) consumo, (7) introducción exitosa, (8) buenos resultados, (9) aprovechamiento como ornamental y forraje, (10) en policultivos, (11) cultivo y exportación, (12) pesca deportiva, (13) piscicultura rural, (14) introducción no exitosa, (15) ornato; procedencia: AFC (África Central), ASI (Asia), ATO (Atlántico Oriental), AMA (Amazonas), BRA (Brasil), BIR (Birmania), CHI (China), COC (Caribe Occidental-Trinidad, Surinam), CEN (Centroamérica), GUA (Guatemala), HAW (Hawái), IND (India), ING (Inglaterra), INP (Indo-Pacífico), MEX (México), PAN (Panamá), PAR (Paraguay), SRI (Sri Lanka), SUM (Sumatra), TAI (Tailandia), BEN (Bengala), USA (Estados Unidos), VZL (Venezuela), (16) accidental.

Tabla 2. Hibridos de tilapia introducidos y producidos en Colombia, con fines de acuicultura intensiva y extensiva. *

\begin{tabular}{|c|c|c|c|}
\hline ESPECIES & $\begin{array}{c}\text { NOMBRE } \\
\text { VERNACULAR }\end{array}$ & INTRODUCIDA & PRODUCIDA \\
\hline Oreocromis (Saroterodon) mossambicus & $\begin{array}{l}\text { Albina, tilapia } \\
\text { roja singapur }\end{array}$ & $X$ & \\
\hline Oreocromis (Saroterodon) niloticus & $\begin{array}{l}\text { Roja, tilapia roja } \\
\text { egipcia }\end{array}$ & $X$ & \\
\hline Oreocromis (Saroterodon) niloticus & $\begin{array}{c}\text { Roja, tilapia roja } \\
\text { ghanesa }\end{array}$ & $\mathrm{X}$ & \\
\hline Oreocromis (Saroterodon) niloticus & $\begin{array}{l}\text { Roja, tilapia roja } \\
\text { tailandesa }\end{array}$ & $\bar{X}$ & \\
\hline $\begin{array}{l}\text { O. mossambicus } \times 0 \text {. niloticus } \times 0 \text {. } \\
\text { aureus }\end{array}$ & $\begin{array}{l}\text { Red aurea, } \\
\text { tilapia roja } \\
\text { israelí }\end{array}$ & & $X$ \\
\hline $\begin{array}{l}0 . \text { mossambicus } \times 0 \text {. urolepis }-0 \text {. } \\
\text { hornorum } \times 0 \text {. niloticus } \times 0 \text {. aureus }\end{array}$ & $\begin{array}{l}\text { Red acci, tilapia } \\
\text { roja colombiana }\end{array}$ & & $X$ \\
\hline $\begin{array}{l}\text { 0. mossambicus } \times 0 \text {. urolepis - } 0 \text {. } \\
\text { hornorum }\end{array}$ & $\begin{array}{l}\text { Red florida, } \\
\text { tilapia roja } \\
\text { floridiana }\end{array}$ & & $X$ \\
\hline O. mossambicus $\times 0$. niloticus & $\begin{array}{c}\text { Red taiwán, } \\
\text { tilapia roja } \\
\text { taiwanesa }\end{array}$ & & $X$ \\
\hline $\begin{array}{l}\text { O. mossambicus } \times 0 \text {. urolopis }-0 \text {. } \\
\text { homorum } \times 0 \text {. niloticus }\end{array}$ & $\begin{array}{c}\text { Red yumbo, } \\
\text { tilapia roja } \\
\text { colombiana }\end{array}$ & & $\bar{X}$ \\
\hline 0. niloticus $\times 0$. mossambicus & $\begin{array}{c}\text { Red sterling, } \\
\text { tilapia roja } \\
\text { sterling, } \\
\text { swansea } \\
\text { mutante }\end{array}$ & & $X$ \\
\hline O. niloticus $\times 0$. aureus & $\begin{array}{c}\text { Tilapia roja, } \\
\text { tilapia roja } \\
\text { israelí }\end{array}$ & & $\mathrm{X}$ \\
\hline $\begin{array}{l}0 . \text { urolepis hornorum } \times 0 . \text { mossambicus } \\
\times 0 \text {. niloticus }\end{array}$ & $\begin{array}{c}\text { Red } \\
\text { nacional,tilapia } \\
\text { roja yumbo }\end{array}$ & & $\mathrm{X}$ \\
\hline
\end{tabular}




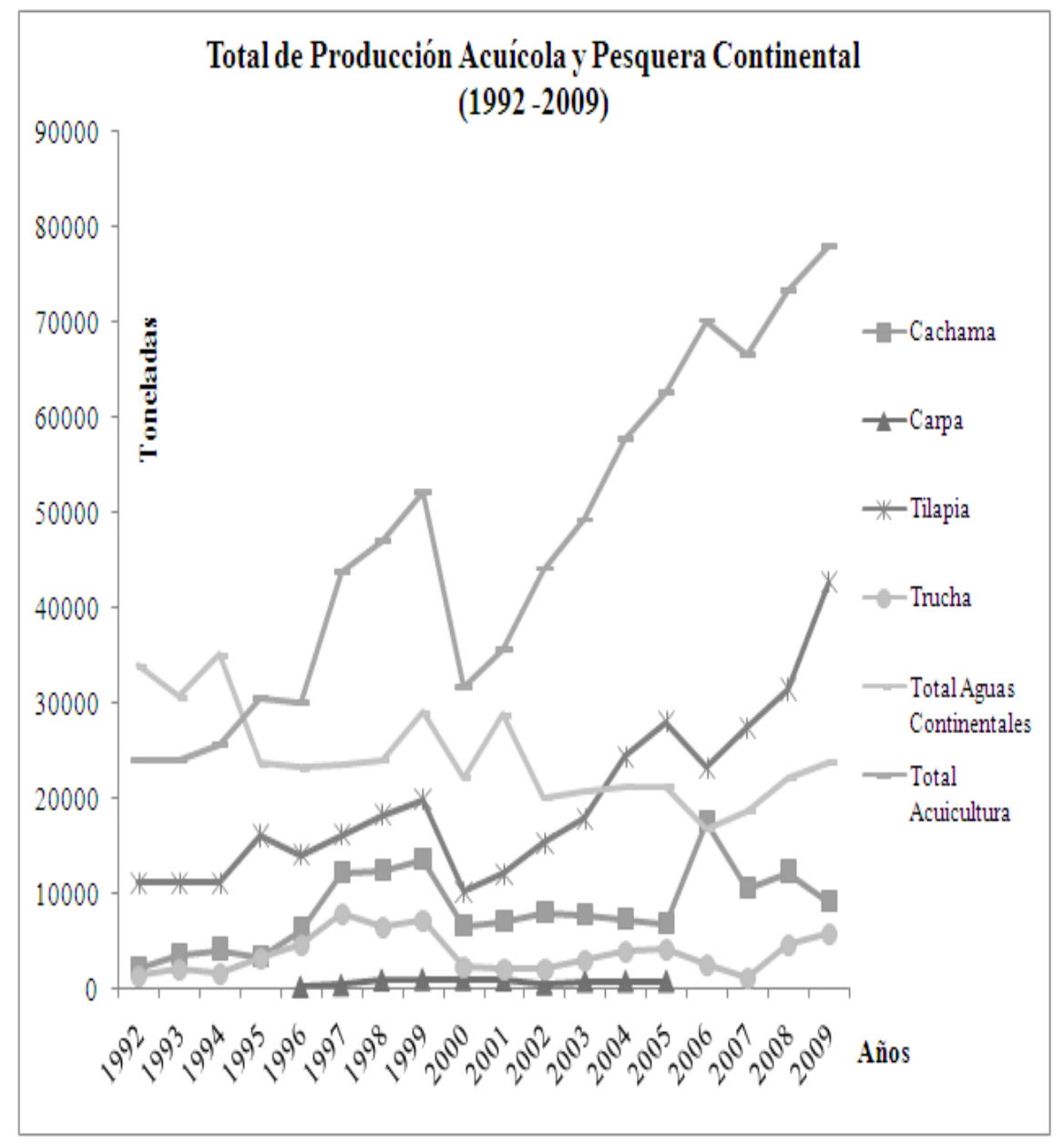

Figura 1. Estadísticas pesqueras del INPA (1992-2002), del INCODER y del ICA (2003-2009). 